\title{
An Investigation of the Histopathological Pattern of Thyroid in Patients Undergoing Thyroid Operations: A Cross-Sectional Study
}

This article was published in the following Dove Press journal: Open Access Surgery

\author{
Saleh Al-wageeh (D) \\ Faisal Ahmed (iD) ${ }^{2}$ \\ Hossein-Ali Nikbakht $\mathbb{D D}^{3}$ \\ Ebrahim Al-shami ${ }^{2}$ \\ Mohammad Reza Askarpour (iD ${ }^{4}$ \\ Umayir Chowdhury (iD ${ }^{5}$ \\ 'Department of General Surgery, lbb \\ University of Medical Science, lbb, \\ Yemen; ${ }^{2}$ Urology Research Center, Al- \\ Thora General Hospital, Department of \\ Urology, lbb University of Medical \\ Science, lbb, Yemen; ${ }^{3}$ Social \\ Determinants of Health Research Center, \\ Health Research Institute, Babol \\ University of Medical Sciences, Babol, \\ Iran; ${ }^{4}$ Department of Urology, Shiraz \\ University of Medical Sciences, Shiraz, \\ Iran; ${ }^{5}$ School of Medicine, Shiraz \\ University of Medical Sciences, Shiraz, \\ Iran
}

Background: Thyroid diseases are major health problems in our society, which are manifested by alteration in hormone secretion, enlargement of the thyroid gland, or both. This study was designed to determine thyroid histopathological patterns in patients who underwent thyroid operation.

Materials and Methods: This retrospective study was carried out at Sana'a University Hospital over two years, from January 1, 2014, to December 30, 2015. All 260 patients who underwent thyroid surgery were included in this study. Factors such as age, sex, residency, fine needle aspiration biopsy (FNAB) result, and post-operative histopathological patterns were recorded. Patients who only underwent FNA were excluded.

Results: The mean age of participants was $40.06 \pm 13.18$ years. Females were most commonly affected by thyroid diseases $(90 \%)$ in comparison to males $(10 \%)$. Histopathologic patterns were nodular colloid goiter $(63.1 \%)$, adenoma $(4.6 \%)$, papillary thyroid neoplasms (20.4\%), follicular thyroid carcinoma (3.8\%), medullary thyroid carcinoma (1.9\%), thyroiditis $(5.4 \%)$ and anaplastic thyroid carcinoma $(0.8 \%)$. Papillary thyroid cancer was the most common thyroid cancer, with a prevalence of 53 (20.4\%). The accuracy of FNAB and final histopathology in combine was $88.07 \%$. However, FNAB was not precise enough in the preoperative diagnosis of follicular carcinoma (40\%). FNAB sensitivity ranged from $70.8 \%$ to $88.8 \%$, while FNAB specificity ranged from $97.1 \%$ to $99.9 \%$.

Conclusion: Nodular colloid goiter is the most prevalent thyroid disease, while papillary thyroid carcinoma is the most frequent cancer seen in this study. We recommend further studies with a larger population to validate our study.

Keywords: goiter, histopathology, papillary carcinoma, thyroidectomy

\section{Introduction}

Thyroid diseases are major health problems, that manifested by alteration in hormone secretion, enlargement of the thyroid gland (goiter), or both. The most prevalent thyroid diseases are goiter, hypo or hyperthyroidism, thyroiditis, and neoplasms. The incidence and prevalence of thyroid diseases in a community are variables which depends on various factors. ${ }^{1,2}$

Thyroid cancers represent approximately $1 \%$ of diagnosed new cancer cases each year. Approximately 23,500 thyroid cancer cases are diagnosed each year in the United States of America (USA). The incidence of thyroid malignancies is three times higher in women than men, and the incidence of thyroid cancer peaks in the third and fourth decades of life. The increased incidence of thyroid cancer is
Correspondence: Faisal Ahmed

Tel +967776089579

Email fmaaa2006@yahoo.com 
associated with an increase in the diagnosis of subclinical thyroid cancers thanks to the development of imaging methods, increased number of patients underwent fineneedles aspiration biopsy (FNAB), more widespread application of total thyroidectomy over subtotal thyroidectomy, and increased precision in the examination of pathological specimens. $^{3,4}$

Thyroid cancers are divided into papillary carcinomas, follicular carcinomas, and medullary thyroid carcinomas (MTCs), anaplastic carcinomas, primary thyroid lymphomas, and primary thyroid sarcomas. To diagnose different kinds of thyroid disease, complete clinical examination in addition to hormonal assays and morphology study should be done. In the end, Histopathologic examination provides a definitive diagnosis. ${ }^{5,6}$ Previous study estimated that thyroid cancer would be the third most common cancer among women in 2019 in the USA. ${ }^{6}$ This cross-sectional study was conducted to identify thyroid disease histopathological patterns in patients who underwent thyroid operations in our center.

\section{Materials and Methods}

\section{Data Inclusion}

This study was conducted in Al-Thawra Modern General Hospital, Sana'a, Yemen, and all patients visiting the surgical out clinic for thyroid diseases, which underwent an operation from January 1, 2014, to December 30, 2015 were included in this study. Those patients in whom fine needle aspiration (FNAB) was done but did not undergo thyroid surgery were excluded from the study. FNAB was carried out in patients having dominant or solitary nodules. All patients had preoperative indirect laryngoscopy to exclude pre-existing vocal cord pathology. Additionally, the accuracy of FNAB was checked using final pathology reports.

\section{Data Gathering}

All biopsy specimens were fixed in formalin and embedded in paraffin sections and stained using Haematoxylin and Eosin (H\&E). All reports, slides, and blocks were kept in the department archives. All sections were diagnosed and reported by pathologists. Information collected includes patients' age, location, sex, FNAB result, histopathologic diagnosis, type of surgery, and complications of surgery. Thyroid diseases were classified based on histological grounds into nodular colloid goiter, adenoma, thyroiditis, and carcinoma, including subtypes of follicular, papillary, medullary, and anaplastic carcinomas.

\section{Statistical Analysis}

The mean \pm SD was used to describe the quantitative variables, while frequency (percent) was used for qualitative variables. Sensitivity and specificity were calculated using standard statistical formulas. The data were analyzed by statistical software SPSS version 20.

\section{Result}

Overall, 260 patients met our criteria during the study period, in which the mean age was $40.06 \pm 13.18$ years. The youngest patient was 11 years old, and the oldest one was 76 years. Females were mostly affected by thyroid diseases (90\%) than males (10\%) with female to male ratio 9:1.

FNAB report was divided to nodular colloid goiter (150 cases, 57.7\%), papillary thyroid neoplasms (50 cases, $19.2 \%$ ), adenoma (22 cases, $8.5 \%$ ), follicular thyroid carcinoma (4 cases, $1.5 \%$ ), thyroiditis (12 cases, $4.6 \%$ ) and anaplastic thyroid cancer (4 cases, 1.5\%). Although 18 cases had an unsatisfactory smear (Table 1 ).

Different types of operation were total thyroidectomy in 117 (45\%) of cases, Hemithyroidectomy in 59 (22.7\%) of cases, Total thyroidectomy with central cervical LN dissection in $50(19.2 \%)$ of cases, and subtotal thyroidectomy in $34(13.1 \%)$ of cases.

A complication of surgery was transient hypocalcemia in $4(1.5 \%)$ of cases, hoarseness in $2(0.8 \%)$ of cases, stridor in $2(0.8 \%)$ of cases, wound infection in $3(1.2 \%)$

Table I Features of All Patients

\begin{tabular}{|c|c|}
\hline \multicolumn{2}{|l|}{ Gender (\%) } \\
\hline Male & $26(10 \%)$ \\
\hline Female & $234(90 \%)$ \\
\hline Age (mean $\pm S D$ ) & $40.06 \pm 13.18$ (years) \\
\hline \multicolumn{2}{|l|}{ Living area (\%) } \\
\hline Urban & $104(40 \%)$ \\
\hline Rural & $156(60 \%)$ \\
\hline \multicolumn{2}{|l|}{ FNAB (\%) } \\
\hline Nodular colloid goiter & $150(57.7 \%)$ \\
\hline Adenoma & $22(8.5 \%)$ \\
\hline Papillary thyroid neoplasms & $50(19.2 \%)$ \\
\hline Follicular thyroid neoplasms & $4(1.5 \%)$ \\
\hline Anaplastic thyroid neoplasms & $4(1.5 \%)$ \\
\hline Thyroiditis & $12(4.6 \%)$ \\
\hline Unsatisfactory smear & $18(6.9 \%)$ \\
\hline
\end{tabular}

Abbreviations: FNAB, fine needle aspiration biopsy; SD, standard deviation. 
Table 2 Post-Operative Complications

\begin{tabular}{|l|c|}
\hline Transient hypocalcemia & $4(\mathrm{I} .5 \%)$ \\
Hoarseness & $2(0.8 \%)$ \\
Strider & $2(0.8 \%)$ \\
Wound infection & $3(1.2 \%)$ \\
Esophageal injury & $\mathrm{I}(0.4 \%)$ \\
Tracheomalacia & $\mathrm{I}(0.4 \%)$ \\
\hline
\end{tabular}

of cases, esophageal injury in $1(0.4 \%)$ of cases, and tracheomalacia in $1(0.4 \%)$ of cases (Table 2$)$.

The histopathology result was nodular colloid goiter in $164(63.1 \%)$ of cases, Adenoma in $12(4.6 \%)$ of cases, papillary thyroid neoplasms in $53(20.4 \%)$ of cases, follicular thyroid carcinoma in $10(3.8 \%)$ of cases, medullary thyroid carcinoma in $5(1.9 \%)$, thyroiditis in $14(5.4 \%)$ of cases, and anaplastic thyroid carcinoma in $2(0.8 \%)$ of cases (Table 3).

Patients were mostly between age 20 to 49 with 202 number of cases in this range (77.7\%) (Table 4). Most of represented cases was from Sana'a, Ibb, Taiz, Hajja, Dahmer, Hudida, Rada, Amran, Albaida, Almahuiet, Raymah with number of cases 102 (39.2\%),44 (16.9\%), 20 (7.7\%), 22 (8.5\%), 18 (6.9\%), 14 (5.4\%), 14 (5.4\%), 8 (3.1\%), $6(2.6 \%), 6(2.3 \%), 6(2.3 \%)$ respectively. $60 \%$ of the cases were from rural areas. In our study, papillary thyroid cancer represents the most common thyroid cancer, representing about 53 cases (20.4\%). FNAB accuracy overall was $88.07 \%$. Accuracy ranged from $100 \%$ in adenoma and anaplastic carcinoma of the thyroid gland. More than $85 \%$ is in papillary, medullary carcinoma of the thyroid gland and thyroiditis, and $91.5 \%$ for nodular colloid. Accuracy could be $40 \%$ in the pre-operative diagnosis of follicular carcinoma.

The sensitivity of FNAB ranged from 70.8 to 88.8 $(81.4 \%)$, and specificity ranged from 97.1 to 99.9 (99.5\%). PPV (Positive predictive value) ranged from 90.9 to $99 .^{7}$ (98.3\%), and NPV (negative predictive

Table 3 Histopathological Patterns of Thyroid Diseases Encountered in 260 Thyroidectomy Specimens Examined

\begin{tabular}{|l|l|}
\hline Nodular colloid goiter & $164(63.1 \%)$ \\
Adenoma & $12(4.6 \%)$ \\
Papillary thyroid neoplasms & $53(20.4 \%)$ \\
Follicular thyroid neoplasms & $10(3.8 \%)$ \\
Medullary thyroid neoplasms & $5(1.9 \%)$ \\
Thyroiditis & $14(5.4 \%)$ \\
Anaplastic thyroid neoplasms & $2(0.8 \%)$ \\
\hline
\end{tabular}

value) ranged from 89.3 to96.2 (93.6\%). Thus, a negative result can mostly rule out the diagnosis of thyroid carcinoma (Table 5).

\section{Discussion}

Diseases of the thyroid gland are a major health problem in our country, and the incidence and prevalence of these thyroid diseases in a community depend on various factors. Tsegaye and associations in 2013 evaluated the histopathologic pattern of thyroid diseases and concluded that Nodular colloid goiter was the most prevalent thyroid disease. ${ }^{1}$ In the same manner, our study showed that Nodular colloid goiter (NCG) is the most prevalent thyroid disease. Previous reports from the west suggest that simple goiter be the most common thyroid disorder that particularly occurs in young women in their childhood and 20 s. Nodular goiter is highly prevalent in iodine-deficient areas. ${ }^{7}$ A higher percentage of nodular goiter in the current study may suggest the possibility of iodine deficiency in representative areas in our study. Further investigations may be required to find causes and pathogenesis in our population.

The female preponderance of the thyroid diseases over males was observed in this study making 9:1 female to male ratio, which is slightly higher, compared to that reported in the literature ranging from $2: 1$ to $9: 1,{ }^{8,9}$ while it is twice the ratio in studies that were conducted in other countries such as Addis Ababa, ${ }^{1}$ Kenya, ${ }^{8,10}$ Ethiopia. ${ }^{11,12}$

In our study, thyroid diseases, particularly NCG, were seen in almost all age groups, mostly in the age group 20-49 years which was similar to surgical textbooks. ${ }^{5,13}$

Our study was limited to 11 cities, mostly from Sana'a, Ibb, Taiz and Hajja respectively, this is mostly due to lack of diagnostic facilities in other areas and proximity of other areas to central hospitals.

The geographic distribution of disease in our study mostly affected the people who live in rural areas that confirm previous similar findings in literature and surgical textbooks. ${ }^{1,14-16}$

FNAB has become the single most important test in the evaluation of thyroid masses and can be performed with or without ultrasound guidance. ${ }^{17}$ The benign thyroid diseases diagnosed by FNAB in the present study were $66.2 \%$, different from previously reported findings in studies United Arab Emirates and Pakistan, respectively. ${ }^{17,18}$ This could be interpreted as the total number of cases, and the population was different in those studies. The malignant thyroid diseases diagnosed by FNAB in the present 
Table 4 The Age Distribution and Histological Categories of 260 Thyroidectomy Specimens

\begin{tabular}{|l|l|l|l|l|l|l|l|l|}
\hline \multicolumn{2}{|l|}{} & \multicolumn{3}{l}{ Age Groups (Year) n (\%) } & \multicolumn{2}{l|}{ Total Number } \\
\cline { 2 - 9 } & $\mathbf{0 - 1 9}$ & $\mathbf{2 0 - 2 9}$ & $\mathbf{3 0 - 3 9}$ & $\mathbf{4 0 - 4 9}$ & $\mathbf{5 0 - 5 9}$ & $\mathbf{6 0 +}$ \\
\hline Pathology report n (\%) & Nodular colloid goiter & $6(3.7)$ & $26(15.9)$ & $44(26.8)$ & $60(36.6)$ & $18(11.0)$ & $10(6.1)$ & 164 \\
& Adenoma & $2(16.7)$ & $2(16.7)$ & $3(25.0)$ & $3(25.0)$ & $0(0.0)$ & $2(16.7)$ & 12 \\
& Papillary & $2(3.8)$ & $9(17.0)$ & $15(28.3)$ & $16(30.2)$ & $3(5.7)$ & $8(15.1)$ & 53 \\
& Follicular & $0(0.0)$ & $2(20.0)$ & $2(20.0)$ & $2(20.0)$ & $0(0.0)$ & $4(40.0)$ & 10 \\
& Anaplastic & $0(0.0)$ & $0(0.0)$ & $0(0.0)$ & $0(0.0)$ & $0(0.0)$ & $2(100.0)$ & 2 \\
& Thyroiditis & $0(0.0)$ & $2(14.3)$ & $6(42.9)$ & $6(42.9)$ & $0(0.0)$ & $0(0.0)$ & 5 \\
& Medullary & $0(0.0)$ & $2(40.0)$ & $2(40.0)$ & $0(0.0)$ & $1(20.0)$ & $0(0.0)$ & 2 \\
\cline { 2 - 10 } & Total & 10 & 43 & 72 & 87 & 22 & 26 & 260 \\
\hline
\end{tabular}

study were higher than previous studies, ${ }^{17,18}$ this might be due to the increasing incidence of malignancies in our country and increasing facilities to diagnose these diseases. In addition, the prevalence of adenoma in our study is incredibly lower than previously reported studies such as Gitau and Hill et al. ${ }^{8,10}$

Carcinomas of the thyroid are relatively uncommon. Most cases occur in adults, although some forms, particularly papillary carcinoma may present in childhood. A female preponderance has been noted among patients developing thyroid carcinoma in the early and middle adult years, and most thyroid carcinomas are well-differentiated types. Exposure to ionizing radiation is considered to be the major predisposing risk factor for thyroid cancer, especially during the first two decades of life. Certain thyroid diseases such as nodular colloid goiter and autoimmune thyroid diseases (Hashimoto's thyroiditis) have been implicated as predisposing factors. Unlike the belief that thyroid carcinoma is a rare disease, ${ }^{13}$ it was seen in $70(28.1 \%)$ cases in our study. Another study reported $23.3 \%$ of thyroid carcinomas. ${ }^{10}$

Thyroid cancer is the most common endocrine malignancy, and in the United States, thyroid cancer accounts for $<1 \%$ of all malignancies ( $2 \%$ of women and $0.5 \%$ of men) and is rapidly increasing in women. ${ }^{19}$ The annual incidence of thyroid cancer varies considerably based on factors such as geographic area, age and sex. ${ }^{13}$ The age distribution of the thyroid carcinoma seen in our study was consistent with surgical textbooks. ${ }^{5,13}$

There were four variants of thyroid carcinoma based on histology, such as Papillary, Follicular, Medullary, and Anaplastic thyroid neoplasms. Papillary thyroid neoplasms account for $80 \%$ of all thyroid malignancies. ${ }^{20}$ In our study, Papillary carcinoma was found to account for

Table 5 The Accuracy Between FNAB and Final Pathology

\begin{tabular}{|c|c|c|c|c|c|c|c|c|c|}
\hline \multicolumn{2}{|c|}{ Final Pathology } & \multicolumn{8}{|c|}{ FNAB n (\%) } \\
\hline & & \multirow{2}{*}{$\begin{array}{l}\text { Nodular } \\
\text { Colloid } \\
\text { Goiter } \\
150(91.5)\end{array}$} & \multirow{2}{*}{$\begin{array}{l}\text { Adenoma } \\
4(2.4)\end{array}$} & \multirow{2}{*}{$\begin{array}{l}\text { Papillary } \\
\text { I }(0.6)\end{array}$} & \multirow{2}{*}{$\begin{array}{l}\text { Follicular } \\
0(0.0)\end{array}$} & \multirow{2}{*}{$\begin{array}{l}\text { Anaplastic } \\
0(0.0)\end{array}$} & \multirow{2}{*}{$\begin{array}{l}\text { Thyroiditis } \\
0(0.0)\end{array}$} & \multirow{2}{*}{$\begin{array}{l}\text { Unsatisfactory } \\
9(5.5)\end{array}$} & \multirow{2}{*}{$\begin{array}{l}\text { Total } \\
164\end{array}$} \\
\hline $\begin{array}{l}\text { Result of } \\
\text { biopsy }\end{array}$ & $\begin{array}{l}\text { Nodular } \\
\text { colloid goiter }\end{array}$ & & & & & & & & \\
\hline n (\%) & Adenoma & $0(0.0)$ & $12(100.0)$ & $0(0.0)$ & $0(0.0)$ & $0(0.0)$ & $0(0.0)$ & $0(0.0)$ & 12 \\
\hline & Papillary & $0(0.0)$ & $4(7.5)$ & $49(92.5)$ & $0(0.0)$ & $0(0.0)$ & $0(0.0)$ & $0(0.0)$ & 53 \\
\hline & Follicular & $0(0.0)$ & $2(20.0)$ & $0(0.0)$ & $4(40.0)$ & $2(20.0)$ & $0(0.0)$ & $2(20.0)$ & 10 \\
\hline & Anaplastic & $0(0.0)$ & $0(0.0)$ & $0(0.0)$ & $0(0.0)$ & $2(100)$ & $0(0.0)$ & $0(0.0)$ & 2 \\
\hline & Thyroiditis & $0(0.0)$ & $0(0.0)$ & $0(0.0)$ & $0(0.0)$ & $0(0.0)$ & $12(85.7)$ & $2(14.3)$ & 14 \\
\hline & Medullary & $0(0.0)$ & $0(0.0)$ & $0(0.0)$ & $0(0.0)$ & $0(0.0)$ & $0(0.0)$ & $5(100)$ & 5 \\
\hline & Total & 150 & 22 & 50 & 4 & 4 & 12 & 18 & 260 \\
\hline
\end{tabular}

Abbreviation: FNAB, fine needle aspiration biopsy. 
(75\%) of thyroid carcinomas. Follicular carcinomas account for $10 \%$ of thyroid cancers and occur more commonly in iodine-deficient areas. Our study shows a slightly higher incidence of follicular carcinoma (14.2\%), which complied with surgical textbooks. ${ }^{5,13}$ Medullary thyroid cancer accounts for about $5 \%$ of all thyroid malignancies and arises from the parafollicular cells of the thyroid. ${ }^{21}$ in our study, we found 5 cases $(7.14 \%)$, which are slightly higher than what was reported in the previous article. ${ }^{21}$ However, it is similar to other published studies. ${ }^{10,22}$ Anaplastic carcinoma accounts for approximately 1\% of all thyroid malignancies in the $\mathrm{USA}^{23}$ but our study showed $2.85 \%$. This difference could be due to the geographical distribution of the disease. The other histological thyroid disease encountered in our study was thyroiditis, with a prevalence of about $5.38 \%$. The low prevalence rate of thyroiditis (3\% and $1 \%$ ) was reported in previous literate. ${ }^{11}$

Previous literates reported that total thyroidectomy is the most appropriate surgical procedure for patients with well-differentiated thyroid carcinoma. Additionally, it is recommended in patients with hyperthyroidism, severe ophthalmopathy, and the previous history of radiation in head and neck regions, large thyroid gland, suspected FNAB, and in goiter with pressure symptoms. ${ }^{24-26}$

Additionally, several studies suggest a total thyroidectomy or Hemithyroidectomy with counter lateral near-total resection to eliminate reoperation due to recurrence and incidental benign cancers. ${ }^{27-29}$ Similarly, most of our operation types were total thyroidectomy and hemithyroidectomy.

In contrast, Matusz et al performed lobectomy in 1088 cases and concluded that lobectomy is a valid alternative to total thyroidectomy for the treatment of papillary thyroid neoplasms patients who are younger than aged 45 years, whose tumor diameter is $4 \mathrm{~cm}$ or less, and who do not have clinical lymph node metastasis or extrathyroidal invasion. ${ }^{30}$

The most common and important complications of thyroidectomy were recurrent laryngeal nerve palsy, hypocalcemia, and hematoma. A systematic meta-analysis review of 14 studies showed 0 to $4 \%$ of transient recurrent laryngeal nerve paralysis, 0 to $2 \%$ of recurrent laryngeal nerve paralysis, 1 to $21 \%$ of transient hypocalcemia, 0 to $2 \%$ of hematoma and overall 0 to $26 \%$ total complication rates had been reported. ${ }^{31}$ In our study, all complications were examined and showed $1.5 \%$ of transient hypocalcemia, $0.8 \%$ hoarseness, $0.8 \%$ stridor, wound infection in $1.2 \%$, esophageal injury in $0.4 \%$, and tracheomalacia in $0.4 \%$ of cases.
Smadi and associations reported that FNAB promises high accuracy at diagnosing papillary, medullary, and anaplastic carcinoma of the thyroid gland, but was not precise enough in the pre-operative diagnosis of follicular carcinoma and Sensitivity ranged from 55 to $90 \%$ and specificity ranged from 90 to $100 \%$. The explanation for lower sensitivity for follicular carcinoma that this cancer is encapsulated and angioinvasive. Our study was in the same line with this result. ${ }^{18}$

The current study had several limitations. First, other thyroid diseases like thyrotoxicosis and hyperthyroidism were not included in our review. Secondly, the sample size of our study limited us from powerful statistical analysis. Thirdly, this is only a retrospective and observational report, which requires validation with larger sample size. Fourthly, there was no access to data on thyroid gland volume and post-operation survival rate. Fifthly, biochemical recurrence, and metastatic recurrence were unavailable in this study.

\section{Conclusion}

Nodular colloid goiter is the most prevalent thyroid disease, while papillary thyroid carcinoma is the most frequent cancer seen in this study. Additionally, performing FNAB initially is more helpful for distinguishing between benign and malignant thyroid nodules. This observation needs to be validated in a larger population study.

\section{Ethics Statement}

The Ethics Committee of Sana'a University of Medical Sciences approved the protocol of the study. Because the data were analyzed anonymously, no informed consent was given. The current study was performed according to the Institutional Committee for the Protection of Human Subjects, which was adopted by the 18th World Medical Assembly, Helsinki, Finland, and its later amendments. The present article was extracted from the thesis written by Saleh Al-wageeh, which was supported by Sana'a University of Medical Sciences.

\section{Author Contributions}

All authors made substantial contributions to conception and design, acquisition of data, or analysis and interpretation of data; took part in drafting the article or revising it critically for important intellectual content; gave final approval of the version to be published; and agree to be accountable for all aspects of the work. 


\section{Disclosure}

The authors declare that they have no competing interests.

\section{References}

1. Tsegaye B, Ergete W. Histopathologic pattern of thyroid disease. East Afr Med J. 2003;80:525-528. doi:10.4314/eamj.v80i10.8755

2. Wortofsky L. Diseases of the thyroid in Fauci, A.S., Braunwald, E. et al ed: Principles of internal medicine. 14th edition; 1998; 2:2012-2035.

3. Davies L, Morris LG, Haymart M, et al. American Association of Clinical Endocrinologists and American College of Endocrinology Disease state clinical review: the increasing incidence of thyroid cancer. Endocr Pract. 2015;21:686-696. doi:10.4158/EP14466. DSCR

4. Gelmini R, Franzoni C, Pavesi E, Cabry F, Saviano M. Incidental thyroid carcinoma (ITC): a retrospective study in a series of 737 patients treated for benign disease. Ann Ital Chir. 2010;81:421-427.

5. Bayliss RIS, Tunbridge WMG. Thyroid Disease: The Facts. USA: Oxford University Press; 1998.

6. Fama F, Sindoni A, Cicciu M, et al. Preoperatively undiagnosed papillary thyroid carcinoma in patients thyroidectomized for benign multinodular goiter. Arch Endocrinol Metab. 2018;62:139-148. doi:10.20945/2359-3997000000017

7. Sushel C, Khanzada TW, Zulfikar I, Samad A. Histopathological pattern of diagnoses in patients undergoing thyroid operations. Rawal Med J. 2009;34:14-16.

8. Gitau W. An analysis of thyroid diseases seen at Kenyatta National Hospital. East Afr Med J. 1975;52:564-570.

9. Mengistu M. A prospective study of 110 Ethiopians with thyrotoxicosis. East Afr Med J. 1992;69:515-519.

10. Hill A, Mwangi I, Wagana L. Thyroid disease in a rural Kenyan hospital. East Afr Med J. 2004;81:631-633. doi:10.4314/eamj. v81i12.9248

11. Mekonen E. Prevalence of goiter in Sekota district, Ethiopia. East Afr Med J. 1996;73:264-267.

12. Mezgebu Y, Mossie A, Rajesh P, Beyene G. Prevalence and severity of Iodine deficiency disorder among children 6-12 years of age in Shebe Senbo District, Jimma Zone, Southwest Ethiopia. Ethiop J Health Sci. 2012;22:196-204.

13. Cotran R, Kumar V. The Thyroid in Robins, SI. Ed. Pathological Bases of Disease. Philadelphia: WB Saunders Company; 1994.

14. Morris LG, Sikora AG, Tosteson TD, Davies L. The increasing incidence of thyroid cancer: the influence of access to care. Thyroid. 2013;23:885-891. doi:10.1089/thy.2013.0045

15. Krukowski ZH. The thyroid and parathyroid glands. In Williams NS, Bulstrode CJK, O Connell PR, eds. Bailey and Love's Short Practice of Surgery. 26th ed. CRC Press, Taylor and Francis Group; 2013:741-77.
16. Townsend T Jr., Beauchamp RD, Evers BM, Mattox KL. Sabiston Textbook of Surgery E-Book: The Biological Basis of Modern Surgical Practice. Elsevier Health Sciences; 2015.

17. Control CfD. Prevention. Improved national prevalence estimates for 18 selected major birth defects-United States, 1999-2001. MMWR. 2006;54:1301.

18. Smadi AA, Ajarmeh K, Wreikat F. Fine-needle aspiration of thyroid nodules has high sensitivity and specificity. RMJ. 2008;33:221-224.

19. Davies L, Welch HG. Increasing incidence of thyroid cancer in the United States, 1973-2002. JAMA. 2006;295:2164-2167. doi:10.1001/ jama.295.18.2164

20. Amdur RJ, Mazzaferri EL. Essentials of Thyroid Cancer Management. New York: Springer; 2005.

21. Haq M, Harmer C. Thyroid cancer: an overview. Nucl Med Commun. 2004;25:861-867. doi:10.1097/00006231-200409000-00001

22. Wahner HW, Cuello G, Correa P, Uribe LF, Gaitan E. Thyroid carcinoma in an endemic goiter area, Cali, Colombia. Amer J Med. 1966;40:58-66. doi:10.1016/0002-9343(66)90187-2

23. Pitt SC, Moley JF. Medullary, anaplastic, and metastatic cancers of the thyroid. Semin Oncol. 2010;37:567-579. Elsevier

24. Liu ZW, Masterson L, Fish B, Jani P, Chatterjee K. Thyroid surgery for Graves' disease and Graves' ophthalmopathy. Cochrane Database Syst Rev. 2015. doi:10.1002/14651858.CD010576.pub2

25. Tezelman S, Borucu I, Senyurek Y, Tunca F, Terzioglu T. The change in surgical practice from subtotal to near-total or total thyroidectomy in the treatment of patients with benign multinodular goiter. World $J$ Surg. 2009;33:400. doi:10.1007/s00268-008-9808-1

26. Giles Y, Boztepe H, Terzioğlu T, Tezelman S. The advantage of total thyroidectomy to avoid reoperation for incidental thyroid cancer in multinodular goiter. Arch Surg. 2004;139:179-182. doi:10.1001/ archsurg.139.2.179

27. Kaliszewski K, Strutyńska-Karpińska M, Zubkiewicz-Kucharska A, et al. Should the prevalence of incidental thyroid cancer determine the extent of surgery in multinodular goiter? PLoS One. 2016;11. doi:10.1371/journal.pone. 0168654

28. Mishra A, Agarwal A, Agarwal G, Mishra S. Total thyroidectomy for benign thyroid disorders in an endemic region. World $J$ Surg. 2001;25:307-310. doi:10.1007/s002680020100

29. Mauriello C, Marte G, Canfora A, et al. Bilateral benign multinodular goiter: what is the adequate surgical therapy? A review of literature. Int J Surg. 2016;28:S7-S12. doi:10.1016/j.ijsu.2015.12.041

30. Matsuzu K, Sugino K, Masudo K, et al. Thyroid lobectomy for papillary thyroid cancer: long-term follow-up study of 1088 cases. World J Surg. 2014;38:68-79. doi:10.1007/s00268-013-2224-1

31. Khadra H, Mohamed S, Hauch A, Carter J, Hu T, Kandil E. Safety of same-day thyroidectomy: meta-analysis and systematic review. Gland Surg. 2017;6:292. doi:10.21037/gs.2017.01.05
Open Access Surgery

\section{Publish your work in this journal}

Open Access Surgery is an international, peer-reviewed, open access journal that focuses on all aspects of surgical procedures and interventions. Patient care around the peri-operative period and patient outcomes post-surgery are key topics for the journal. All grades of surgery from minor cosmetic interventions to major surgical procedures are covered. Novel techniques and the utilization of new

Submit your manuscript here: https://www.dovepress.com/open-access-surgery-journal instruments and materials, including implants and prostheses that optimize outcomes constitute major areas of interest. The manuscript management system is completely online and includes a very quick and fair peer-review system, which is all easy to use. Visit http://www.dovepress.com/testimonials.php to read real quotes from published authors. 